\title{
Use of tissue recombination to predict phenotypes of transgenic mouse models of prostate carcinoma
}

\author{
Kenichiro Ishii ${ }^{1}$, Scott B Shappell ${ }^{1,2,3, *}$, Robert J Matusik ${ }^{1,3,4}$ and Simon W Hayward ${ }^{1,3,4}$ \\ ${ }^{1}$ Department of Urologic Surgery, Vanderbilt University Medical Center, Nashville, TN, USA; ${ }^{2}$ Department of \\ Pathology, Vanderbilt University Medical Center, Nashville, TN, USA; ${ }^{3}$ The Vanderbilt-Ingram Cancer Center, \\ Vanderbilt University Medical Center, Nashville, TN, USA and ${ }^{4}$ Department of Cancer Biology, Vanderbilt \\ University Medical Center, Nashville, TN, USA
}

\begin{abstract}
Transgenic mouse models of cancer represent a powerful approach for exploring disease processes and testing potential therapeutic interventions. Currently, it is difficult to predict if a specific genetic manipulation will result in a desirable phenotype. The present study tests the idea that tissue recombinants recapitulate the pathologic features of the neoplastic prostate seen in transgenic mice, and would thus be suitable predictive models for new mouse design. The large probasin-large T-antigen (LPB-Tag) transgenic lines 12T-7f and 12T-10 were used as a basis for this study. Tissue recombinants of bladder epithelium (BIE) and urogenital sinus mesenchyme (UGM) were implanted under the renal capsule of athymic mice. Recombinants composed of BIE from 12T-10 LPB-Tag and wild-type (wt) UGM faithfully recapitulated the histopathologic and temporal features of intact transgenic mice of this line. Tissue recombinants using BIE from 12T-7f mice and wt UGM developed epithelial proliferation with atypia that lacked the associated hypercellular stroma seen in the intact 12T-7f line. Recombinants using 12T-7f UGM demonstrated that the hypercellular stroma results from stromal cell expression of the SV40 large T antigen. Corresponding to the recombinant phenotypes, stromal Tag immunostaining was observed in prostate tissues from intact 12T-7f but not 12T-10 mice. Similar stromal expression of Tag was also noted in the hypercellular TRAMP prostatic stroma. Further analysis revealed a previously unreported pattern of SV40T expression in the LADY and TRAMP models including ductus deferens and seminal vesicle stroma as well as region and cell type-specific patterns in the epididymis. The present study demonstrates the utility of using tissue recombination to explore organ-specific phenotypes. Recombination strategies should enable quick and cost-effective screening for likely phenotypes in transgenic animals. This comparison of tissue recombination to existing models shows that this approach can elicit new information on well-characterized models.
\end{abstract}

Laboratory Investigation (2005) 85, 1086-1103. doi:10.1038/labinvest.3700310; published online 27 June 2005

Keywords: transgenic; genetically engineered; prostate; TRAMP; LPB-Tag; tissue recombination

Transgenic mice are useful models for investigation of the effects of genetic changes in the development and progression of tumors. A number of different promoters have been used to target transgene expression to the prostate, one of the most successful of which has been the probasin (PB) promoter system. ${ }^{1-4} \mathrm{~PB}$ is a rodent prostatic secretory protein that belongs to the lipocalin ligand carrier protein

Correspondence: Dr SW Hayward, PhD, Department of Urologic Surgery, A1302 MCN, Vanderbilt University Medical Center, Nashville, TN 37232-2765, USA.

E-mail: simon.hayward@vanderbilt.edu

${ }^{*}$ Current address: Molecular Pathology, Oppenheimer Urologic Reference Laboratory, Nashville, TN, USA.

Received 28 February 2005; revised 17 May 2005; accepted 18 May 2005; published online 27 June 2005 superfamily. The expression of $\mathrm{PB}$ is prostate specific and is regulated by androgens and zinc. ${ }^{4-6}$ Genomic analysis demonstrates that $\mathrm{PB}$ is found in a lipocalin cluster on the rodent X-chromosome and that the human syntenic X-chromosomal region is deleted. Six epididymal-specific lipocalins have evolved by in situ tandem duplication resulting in a locus of genes that show highly segment-specific expression patterns in the epididymis with different modes of regulation. ${ }^{7}$ This demonstrates that the preservation of tissue-specific regulatory sequences resides not only in the adjacent promoter but also in a locus control region.

The first generation PB promoter was a 454 base pair fragment, which achieves the lowest level of transgene expression in the mouse prostate, and which has been used in a number of transgenic 
mice, most notably in the transgenic adenocarcinoma of the mouse prostate (TRAMP) model. ${ }^{8} \mathrm{~A}$ second large $\mathrm{PB}$ (LPB) promoter, ${ }^{2,9}$ which contains many more of the natural control and enhancer elements, achieves much higher levels of transgene expression in the mouse prostate and was utilized in the generation of the large $\mathrm{PB}$ promoter-large $\mathrm{T}$ antigen (LPB-Tag) or LADY models of prostate carcinoma (Pca). ${ }^{2,9}$ However, at almost $11 \mathrm{~kb}$ in length, the LPB promoter construct is difficult to use. A third generation $\mathrm{ARR}_{2} \mathrm{~PB}$ promoter was designed to contain additional androgen response elements from the natural $\mathrm{PB}$ promoter in a much shorter piece of DNA, and achieves high levels of prostatic transgene expression. ${ }^{3}$ This current generation of the promoter is used in many contemporary models targeting gene expression to the mouse prostate or to specifically eliminate gene expression in the mouse prostate using Cre-Lox technology. ${ }^{10-15}$

Until recently, models that target the SV40 large T antigen (Tag) have been the only ones that consistently develop unequivocal invasive and potentially metastatic Pca. ${ }^{16}$ As well as occasional examples of well-to-moderately differentiated adenocarcinomas, Tag-incorporating models have been recognized to typically show features of neuroendocrine (NE) carcinoma or poorly differentiated carcinomas with NE differentiation. ${ }^{16}$

The TRAMP model ${ }^{8}$ uses the SV40 early region that expresses both the small and large $\mathrm{T}$ antigens while LPB-Tag $(\mathrm{LADY})^{9}$ uses only the large $\mathrm{T}$ antigen. The LPB-Tag model group comprises several distinct mouse strains including the 12T-7f, $12 \mathrm{~T}-7 \mathrm{~s}, 12 \mathrm{~T}-10$ and 12T-11 models. These models have different sites of transgene integration and exhibit different rates of neoplastic prostate growth paralleled by differences in histopathology. ${ }^{9}$ The fast growing $12 \mathrm{~T}-7 \mathrm{f}$ model forms mouse prostatic intraepithelial neoplasia (mPIN) lesions, characterized by a uniform marked prostatic lobular expansion by neoplastic epithelium, which can progress to unequivocal associated invasive adenocarcinoma. In contrast, 12T-10 mice show a slower prostate growth rate, with the neoplastic epithelial proliferation of the mPIN lesions occurring within more normalappearing gland profiles, and commonly gives rise to invasive and metastatic $\mathrm{NE}$ carcinoma. ${ }^{16-19}$ TRAMP also develops extensive lesions reported to be well-differentiated adenocarcinomas ${ }^{20}$ but showing morphologic overlap with the atypical epithelial proliferations regarded as in situ mPIN as defined by the NCI Mouse Models of Human Cancer Consortium (MMHCC) report $^{16}$ and as so reported in the fast growing LADY lines. ${ }^{8,16,19}$ TRAMP shows progression to extensively invasive NE tumors that have metastatic potential. ${ }^{8,16,19}$ Of the LADY models, the 12T-10 develops a primary NE prostate cancer, which progresses to metastasis. ${ }^{9,16,19,21}$ The 12T-7f, 12T-7 s, and 12T-11 models develop primary tumors that are mPIN with small foci of invasive cancer and rare metastasis.,
However, the $12 \mathrm{~T}-7 \mathrm{f}$ model will progress to adenocarcinoma and develop NE bone metastasis by overexpressing the hepsin gene within this model. ${ }^{22}$

In relation to the present study, stromal hypercellularity is observed to accompany atypical epithelial proliferations in the dorsolateral (DLP) and anterior prostate (AP) of fast growing LPB-Tag lines, such as 12T-7f. ${ }^{16,17,19}$ In contrast, stromal hypercellularity is not noted to any significant degree in the $12 \mathrm{~T}-7 \mathrm{f}$ ventral prostate, nor in any prostatic lobes of the 12T-10 line. ${ }^{9,16,19,21}$ Similar stromal hypercellularity is noted to a somewhat lesser degree in the TRAMP mouse. ${ }^{8,16}$ The stromal hypercellularity in these models has morphologic features in common with the normal contractile fibromuscular prostatic stroma (particularly at earlier time points). Stromal hypercellularity is progressive with age and fairly uniform in nature, which, along with other properties, distinguishes it from a focal desmoplastic response to invasive carcinoma. ${ }^{16} \mathrm{~A}$ similar prostatic stromal proliferation is not a usual or common feature of prostatic epithelial neoplasia in the human. Occasionally, a more condensed stroma, including with cytologic atypia, is seen more focally in closer association with the neoplastic epithelium in models such as LADY and TRAMP. ${ }^{16}$ The nature of such spindle cells, and whether they are related to an epithelial-mesenchymal transformation, or part of the more generalized stromal alterations is not known.

The process of generating and characterizing transgenic and gene knockout mice, while now much simpler and cheaper than in the past, is still time consuming. There are a large number of candidate genetic manipulations that could be employed to model various aspects of the development and progression of human Pca. Therefore, it would be desirable to establish in advance that such models are likely to exhibit a phenotype. The use of tissue recombinants may allow for such an analysis and might also be able to determine the contribution of neoplastic epithelium vs stromal cells to observed phenotypes in intact transgenic mice.

The main objective of the current study was to determine the feasibility of using tissue recombination as a screening tool to establish prostatic phenotypes in advance of generating new transgenic models. As a first step in this endeavor, we tested whether tissue recombinants would faithfully recapitulate the progression of well-characterized models of Pca. For this purpose, we chose the fast growing LADY 12T-7f and the slower 12T-10 model. Urogenital sinus mesenchyme (UGM) is a wellestablished inducer of prostatic differentiation from adult bladder epithelium. ${ }^{23,24}$ In order to accurately control the length of time that the epithelial cells expressed SV40T antigen (and thus examine temporal aspects of tumor development), recombinants in this study utilized transgenic mouse bladder epithelial cells as a starting point in these studies. Our findings confirm the feasibility of the approach 
and raise important issues relating to the specificity of cell type-specific promoters in transgenic animals. Additionally, we were able to shed light on the origins of the hyperproliferative stroma seen in the LADY and TRAMP models of prostate cancer.

\section{Materials and methods}

\section{Transgenic Mouse Tissue}

12T-7f, 12T-7s, 12T-10 and 12T-11 mice were from our own stocks. ${ }^{9,21}$ Mice were killed by cervical dislocation under anesthetic overdose. Prostate (all lobes: ventral, dorsolateral and anterior), seminal vesicle, bladder, kidney, epididymis, ductus deferens, testis, intestine, stomach, liver, spleen, lung, skin (ear), skeletal muscle, heart and brain were harvested from 12-week-old 12T-7f, 12T-7s, 12T-10 and 12T-11 LPB-Tag male mice. For routine histology and immunohistochemical examination all organs were fixed in 10\% neutral-buffered formalin at room temperature overnight and processed to paraffin using standard procedures. Paraffin-embedded tissue blocks of adult C57BL6/FVB TRAMP mice with prostate portions representing the spectrum of neoplastic progression in this model, seminal vesicle and lymph node metastases were supplied by Jackson Laboratories for the 2001 Workshop 'Techniques in modeling human prostate cancer in mice' (courtesy of Dr William Tu, VUMC).

\section{Tissue Separation and Recombination}

Rat UGM (rUGM) was prepared from 18-day embryonic fetuses of pregnant Sprague-Dawley rats (Harlan Sprague Dawley Inc., Indianapolis, IN, USA). Mouse UGM (mUGM) was prepared from 16-day embryonic fetuses of pregnant 12T-7f LPBTag heterozygous mice that were crossed to male CD-1 mice. The genetic identity of individual mouse fetuses was confirmed by PCR genotyping. Dissection and separation of urogenital sinus epithelium (UGE) and UGM was performed as previously described. ${ }^{25}$ Briefly, urogenital sinuses were dissected from fetuses and separated into epithelial and mesenchymal components by a 90-min digestion at $4^{\circ} \mathrm{C}$ with $10 \mathrm{mg} / \mathrm{ml}$ trypsin 1:250 (Sigma Chemical Co., St Louis, MO, USA) followed by mechanical separation. UGM was then additionally reduced to single cells by a 90-min digestion at $37^{\circ} \mathrm{C}$ with $187 \mathrm{U} / \mathrm{ml}$ collagenase (Gibco-BRL, Grand Island, NY, USA). After digestion, the cells were washed extensively with RPMI 1640 . Viable cells were then counted using a hemacytometer, with viability determined by Trypan blue exclusion.

The adult bladder urothelium of wild-type (wt) CD-1 mice, LPB-Tag 12T-7f and LPB-Tag 12T-10 mice were separated from the stroma by a $45-\mathrm{min}$ digestion at room temperature with $20 \mathrm{mM}$ EDTA. $^{23,24}$ Epithelial tissue fragments were then recombined with UGM in neutralized type I rat tail collagen gels as previously described. ${ }^{26}$ Parallel studies also used recombinants composed of rUGM plus epithelial fragments from the DLP of 4- or 12week-old wt or LADY mice. Tissue recombinants were grafted beneath the renal capsule of adult homozygous athymic CD-1 male nude mice (Charles River Laboratories, Wilmington, MA, USA) as described. ${ }^{25}$ Mice were killed and the grafts harvested at different periods after grafting as indicted in the Results section.

\section{Histopathology and Immunohistochemistry}

Sections $(5 \mu \mathrm{m})$ were cut from representative paraffin-embedded samples and were stained with H\&E or utilized for immunohistochemistry. Histopathologic assessment on recombinants utilized similar criteria as well established for prostate of genetically engineered mice in intact animals, including LADY and TRAMP. ${ }^{16}$ For immunohistochemistry, sections were deparaffinized in Histo-Clear (National Diagnostic, Atlanta, GA, USA) and rehydrated in a graded series of ethanol. Endogenous peroxidase was blocked by $0.3 \%$ hydrogen peroxide in methanol for $20 \mathrm{~min}$. After extensive washing in tap water, antigen retrieval was performed using $10 \mathrm{mM} \mathrm{Na}$ citrate buffer, $\mathrm{pH} 6.0$ for $\mathrm{AR}$ and antigen unmasking solution (Vector laboratories Inc., Burlingame, CA, USA) for Tag immunostaining. After rinsing in phosphate-buffered saline (PBS), the sections were incubated in CleanVision ${ }^{\mathrm{TM}}$ blocking solution (ImmunoVision Technologies Co., Daly City, CA, USA) for $30 \mathrm{~min}$ at room temperature to block nonspecific binding. The sections were then incubated with anti-AR antibody N-20 (1:500 dilution) (Santa Cruz Biotechnology, Santa Cruz, CA, USA), anti-Tag antibody Pab 101 (1:100 dilution) (Santa Cruz Biotechnology, Santa Cruz, CA, USA) at $4^{\circ} \mathrm{C}$ overnight. After incubation with primary antibody, sections were incubated with appropriate biotinylated secondary anti-mouse or anti-rabbit immunoglobulin (DakoCytomation, Carpinteria, CA, USA) diluted with PBS at 1:200 for $30 \mathrm{~min}$ at room temperature, respectively. The antigen-antibody reaction was visualized with the Vectastain avidin-biotin complex kit (Vector Laboratories Inc., Burlingame, CA, USA) using 3,3'-diaminobenzidine tetrahydrochloride as a substrate. The sections were counterstained with hematoxylin and examined by a light microscopy.

\section{Results}

Phenotype of Tissue Recombinants Composed of Rat UGM and either LPB-Tag 12T-7f or 12T-10 Bladder Epithelium (BlE)

Control tissue recombinants made with wt BlE and wt rUGM harvested at 4 and 24 weeks exhibited 


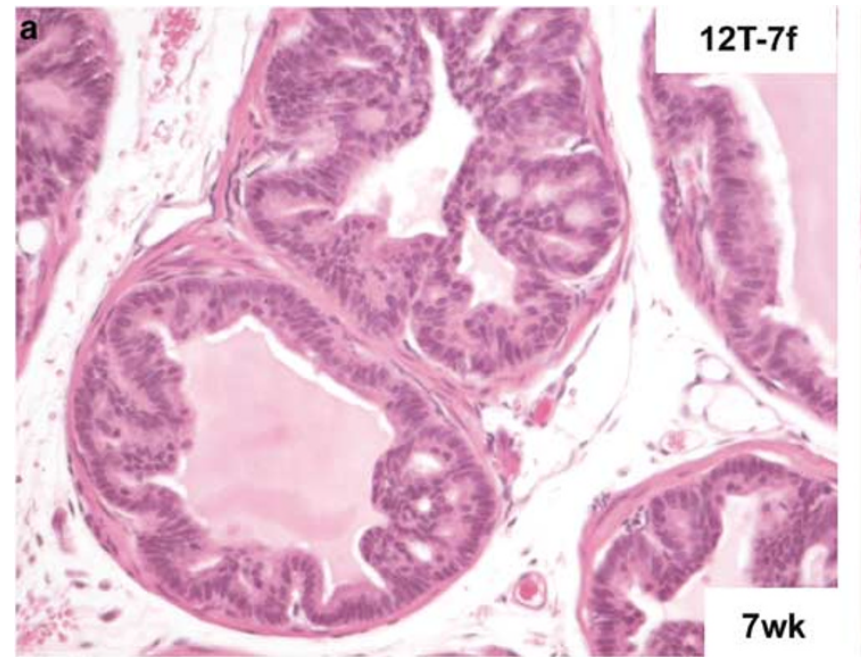

b

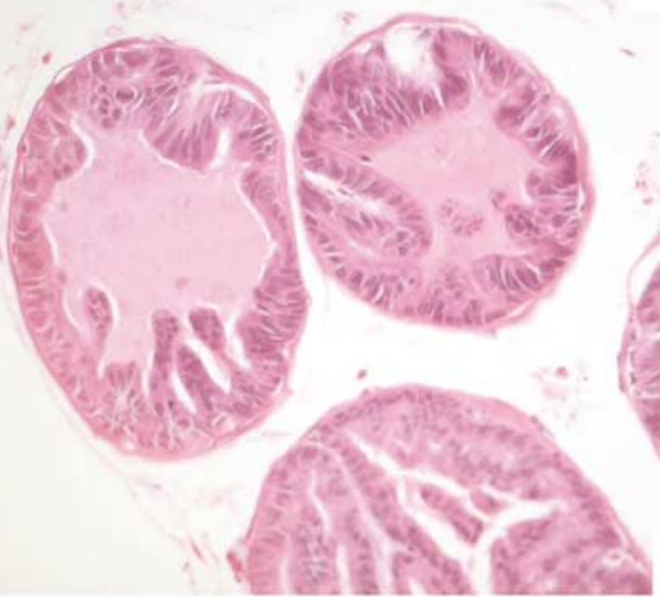

$12 \mathrm{~T}-10$

ve -
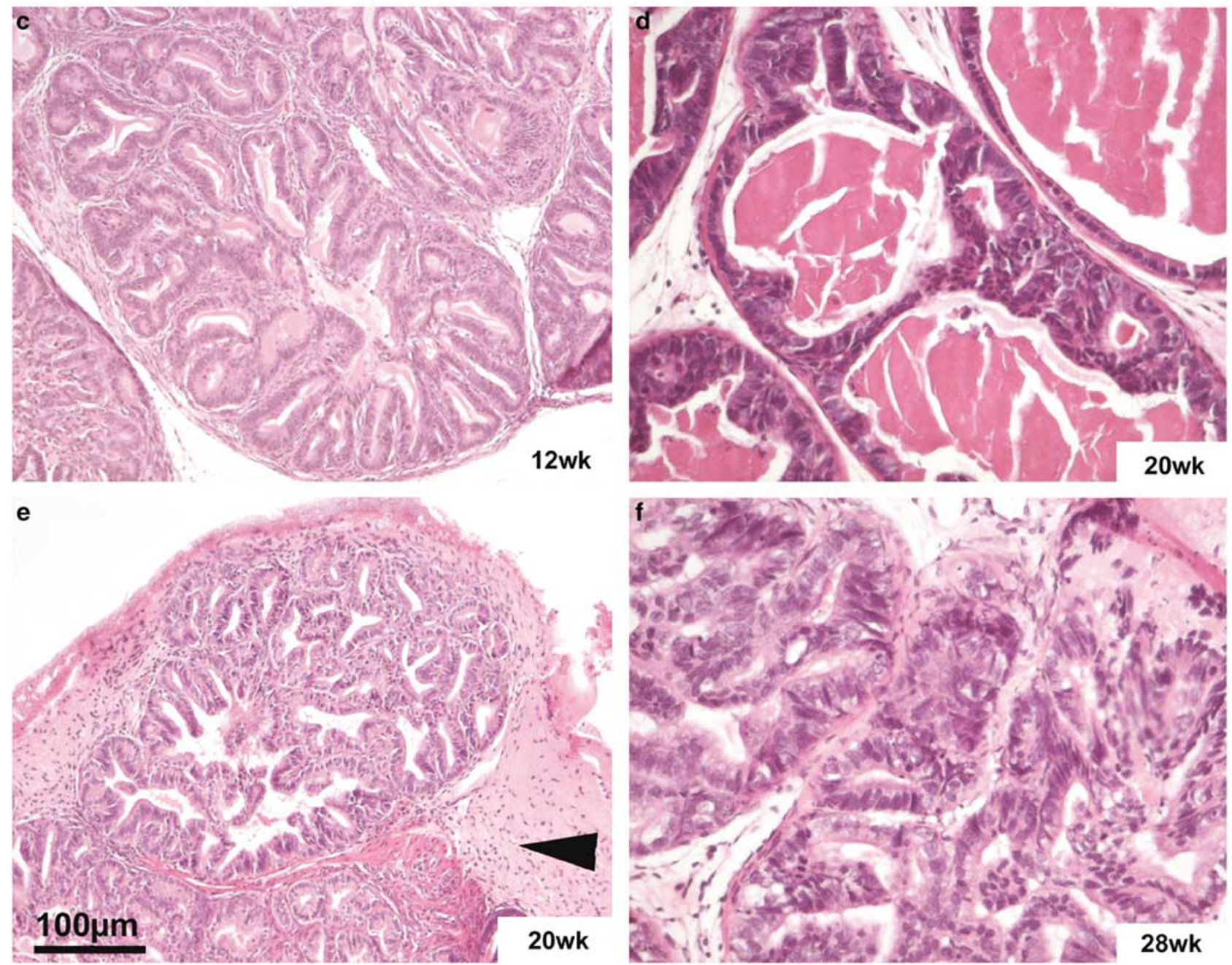

Figure 1 Ontogenic progression of dorsolateral prostatic lesions in 12T-7f and 12T-10 LPB-Tag mice. Progression of the lesions in the $12 \mathrm{~T}-7 \mathrm{f}$ tumors (a, $\mathbf{c}$ and $\mathbf{e}$ ) is more rapid than in the 12T-10 mice (b, $\mathbf{d}$ and $\mathbf{f}$ ). In $12 \mathrm{~T}-7 \mathrm{f}$ mice at 7 weeks of age (a) atypical epithelial proliferation is seen with rims of slightly hypercellular stroma. At 12 weeks (c) progressive expansion of atypical epithelium is seen restricted within the surrounding somewhat hypercellular contractile stroma consistent with mPIN. By 20 weeks of age (e) the mPIN lesion is more complex but definitive invasive adenocarcinoma is rare, a more pronounced hypercellular stroma (arrowhead) is seen at this age. 12T-10 tumors grow more slowly than those seen in 12T-7f animals. At 16 (b) and 20 weeks of age (d) there is a less pronounced lobular expansion of the mPIN lesion with atypical epithelium confined to ducts with more normal architecture. By 28 weeks (f) there is more pronounced architectural disruption, however, the abnormal cells remain constrained within normal-appearing surrounding stroma consistent with an mPIN lesion. 
normal-appearing prostate histology (data not shown). Ducts with normal prostatic secretory differentiation and with an associated thin layer of contractile fibromuscular stroma existed in an orderly lobular pattern similar to intact prostate. These results are consistent with previously published studies. ${ }^{23,24,27,28}$

Tissue recombinants composed of adult $12 \mathrm{~T}-7 \mathrm{f}$ BlE and rUGM were harvested at 3, 4, 6, 8, 12, 24 and 34 weeks postgrafting. These recombinants showed a progressively severe neoplastic epithelial lesion compatible with the development and progression of $\mathrm{mPIN}^{16}$ and similar to that seen in the prostate of intact 12T-7f mice (Figure 1). As in control grafts, these recombinants readily formed prostatic tissue. At early time points there was proliferation of atypical epithelium within prostate ducts/glands which themselves exhibited fairly normal architecture (Figure 2), similar to intact $12 \mathrm{~T}-7 \mathrm{f}$ mouse prostate (Figure 1). These glands were generally surrounded with thin rims of normalappearing contractile fibromuscular stroma. Mild nuclear atypia was manifested by enlargement and hyperchromasia, without prominent stratification as early as 6 weeks postgrafting (Figure 2). An overall increase in glandular components compared to recombinants made with wt epithelium and intact wt prostates was noted. However, small acinar profiles at the periphery of each lobule connected up to the central duct lumen in a symmetric fashion, supporting the interpretation of mPIN rather than invasive adenocarcinoma. ${ }^{16}$ With increasing time postgrafting more pronounced degrees of atypical epithelial proliferation were found (Figure 2). Neoplastic epithelium demonstrated progressively increased nuclear atypia including more pronounced nuclear enlargement, stratification and hyperchromasia. It was notable that the epithelial glands continued to be surrounded by a normalappearing thin rim of stroma. By 12 weeks postgrafting there was a continued expansion of the atypical epithelial compartment, wherein multiple duct profiles show lobular expansion by neoplastic epithelium again with a normal thin rim of surrounding contractile stroma. The connection of smaller gland profiles occasionally seen at the periphery of lobules to more central lumens, and similar atypia in both, with no focal deviation from this general architectural pattern, is quite similar to the epithelial component of the DLP of intact 12T-7f animals. This lesion is regarded by consensus conference as an in situ mPIN neoplasm (Figure 1). This phenotype continued out to 34 weeks postgrafting at which point foci of accentuated microacinar architecture with distinctly accentuated nuclear atypia, with vesicular nuclei and prominent nucleoli were seen (Figure 2). Areas of early potentially invasive adenocarcinoma, distinct from background mPIN, when evaluated according to criteria established by the NCI MMHCC ${ }^{9}$ were seen at 34 weeks postgrafting (Figure 2). Even at this time point the stroma surrounding the ducts appeared normal, in marked contrast to the fairly uniform hypercellular stroma in intact $12 \mathrm{~T}-7 \mathrm{f}$ mouse prostate (Figure 1). AR immunostaining in sections of the 12T-7f BlE + rUGM recombinants showed nuclear staining in the proliferating atypical epithelium as well as in the surrounding spindled stroma cells (Figure 3 and Supplementary Figure 1). Tag immunostaining showed nuclear staining of the proliferating atypical epithelium. In the tissue recombinants, there was no Tag immunoreactivity in the surrounding normal-appearing fibromuscular stroma (Figure 3 and Supplementary Figure 1). At the longest time point investigated (34 weeks), AR and Tag staining were no longer uniform in the epithelium with focal negative staining in areas noted as potentially representing foci of invasion (Figure3 and Supplementary Figure 1).

The results of parallel studies using $12 \mathrm{~T}-7 \mathrm{f}$ prostatic epithelium and rUGM harvested at 4 and 12 weeks postgrafting (data not shown) were essentially identical to the bladder epitheliumbased studies. The atypical epithelial cell component was highly reminiscent of the mPIN lesion in the DLP of intact fast growing 12T-7f transgenic mice while the stroma was essentially normal in appearance.

Similar tissue recombinants were made using $12 \mathrm{~T}$ $10 \mathrm{BlE}$ with rUGM. These were harvested at 16, 20 and 28 weeks postgrafting and exhibited a neoplastic epithelial phenotype similar to that in the prostates of intact 12T-10 mice $^{21}$ (Figure 1). Epithelial cell proliferation was manifested by stratification with prominent nuclear atypia, including nuclear enlargement, hyperchromasia, chromatin

\footnotetext{
Figure 2 Tissue recombinants composed of 12T-7f BlE and rUGM. Intermediate- and high-power photomicrographs, respectively, of H\&E-stained sections of recombinants harvested at 6 (a and $\mathbf{b}$ ), 8 (c and d), 12 (e and $\mathbf{f}$ ) and 34 (g and $\mathbf{h}$ ) weeks postgrafting. (a) At 6 weeks proliferation of atypical epithelium within prostate ducts/glands of fairly normal architecture, with thin rims of normal appearing contractile fibromuscular stroma surrounding ducts. (b) Detail from a, showing mild nuclear atypia in epithelial cells manifested by enlargement and hyperchromasia, without prominent stratification. (c) At 8 weeks more pronounced degrees of atypical epithelial proliferation are seen. (d) Detail from c showing neoplastic epithelium with increased nuclear atypia (eg compare to b), with normal appearing thin rim of stroma. (e) At 12 weeks a progressive expansion of atypical epithelial compartment is seen, wherein multiple duct profiles show lobular expansion by neoplastic epithelium with normal thin rim of surrounding contractile stroma. (f) A detail of e showing marked nuclear atypia similar to the epithelial component of the DLP of intact 12T-7f animals, and consistent with an in situ neoplasm (ie mPIN). (g and h) At 34 weeks similar mPIN lesions, with focal, somewhat distinct area of accentuated microacinar architecture, with distinctly accentuated nuclear atypia shown in detail in (h), with vesicular nuclei and prominent nucleoli, potentially representing an area of early invasive adenocarcinoma, distinct from background mPIN. There is associated fairly normal surrounding stroma, even at this latest time point.
} 

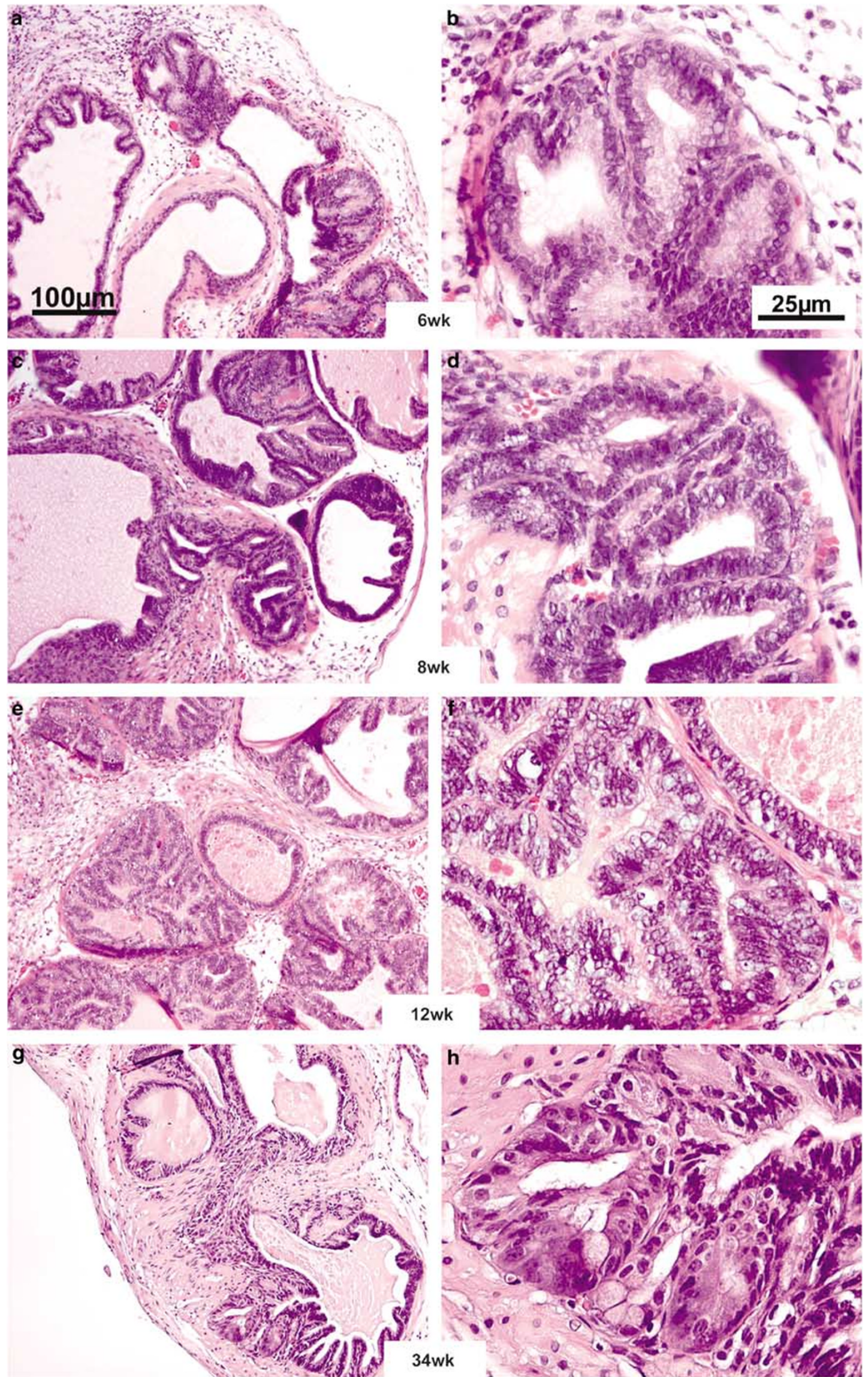
1092
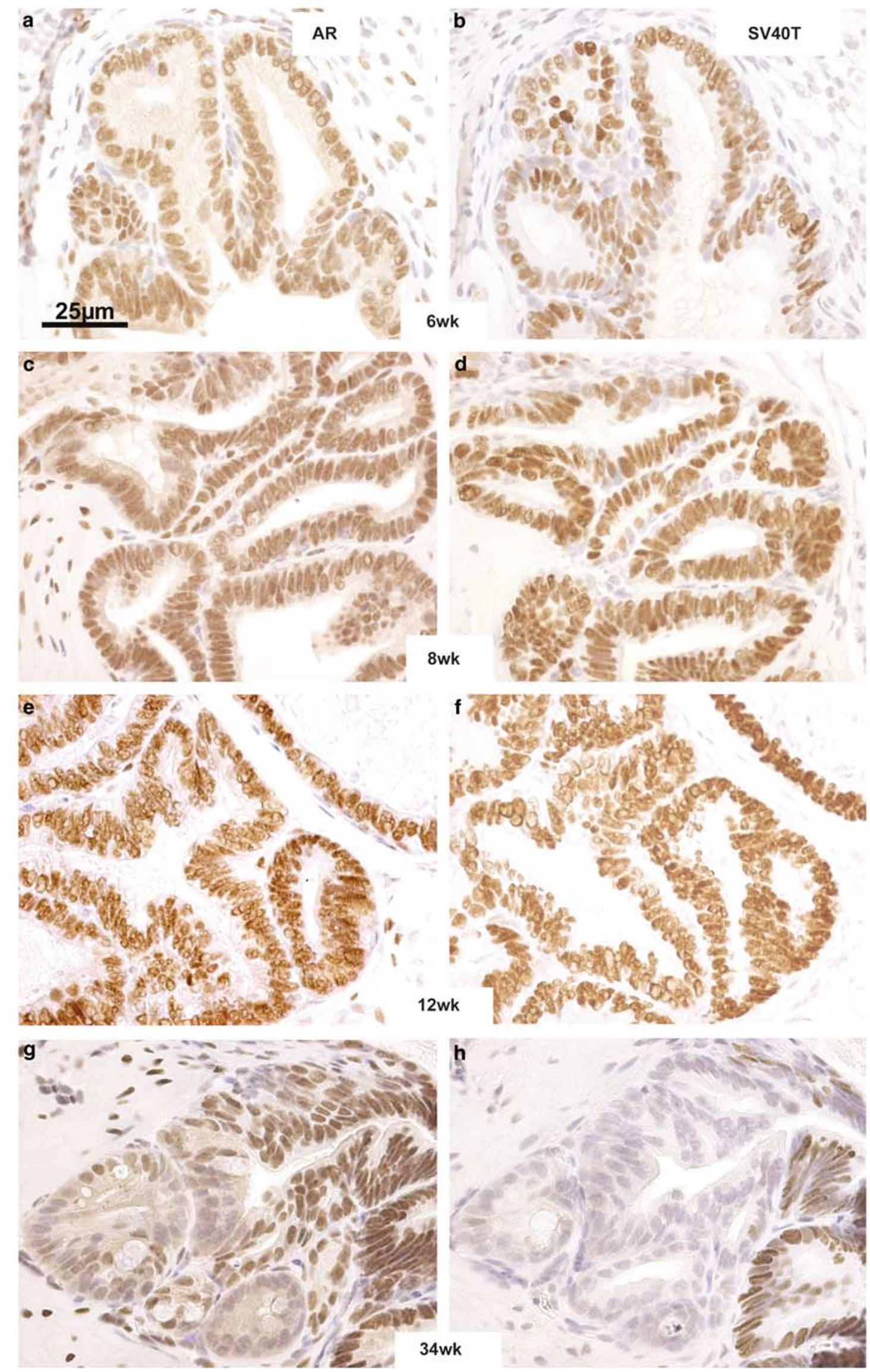
clumping and mitoses (Figure 4). Temporally, these grafts exhibited progressive epithelial proliferation, in terms of both the extent and degree of nuclear atypia, compatible with mPIN in intact $12 \mathrm{~T}-10$ mouse prostate. Particularly at the 16 and 20 weeks time points (Figure 4), the slower development of mPIN lesions as compared to similar recombinants with 12T-7f epithelium (Figure 2) was evident. The less pronounced lobular expansion of the mPIN lesion with the 12T-10 epithelium, with atypical epithelium more confined to ducts with more normal profiles was also similar to the phenotypic differences in intact $12 \mathrm{~T}-10$ vs $12 \mathrm{~T}-7$ mouse prostate (Figure 1). The progression of these changes is compatible with the designation of mPIN in the intact prostate. ${ }^{16}$ In contrast to the mPIN lesions in intact $12 \mathrm{~T}-7 \mathrm{f}$ mice and the $12 \mathrm{~T}-7 \mathrm{f}$ recombinants described above, and similar to the mPIN lesions in intact 12T-10 mice, the neoplastic epithelial proliferation was confined to architecturally normal duct/ gland spaces; that is, of similar size and shape to that of wt mouse prostate and tissue recombinants made with wt bladder or prostate epithelium and rUGM (Figure 4). Also similar to the mPIN lesions in intact 12T-10 mice, the surrounding stroma was of normal histology, composed of a typical thin rim of contractile fibromuscular stroma, without hypercellularity or atypia.

\section{Phenotype of Tissue Recombinants Composed of Wild Type vs 12T-7f Mesenchyme}

To investigate the origin of the hypercellular stroma in the neoplastic prostate of intact LPB-Tag 12T-7f mice, we made each of the four possible tissue recombinants between BlE epithelium and UGM of $12 \mathrm{~T}-7 \mathrm{f}$ or wt mice (Table 1). Recombinants were examined at 4, 8 and 12 weeks postgrafting (Figure 5 and Supplementary Figure 2).

Morphologically, tissue recombinants composed of wt BlE and wt mUGM were essentially similar to the wt BlE and wt rUGM controls described above. Prostatic ducts in recombinants had an arrangement similar to that in the intact mouse prostate. Epithelial cells showed normal secretory differentiation, similar to that of the DLP of intact wt mouse prostates. Ducts were lined with cuboidal to columnar epithelium containing round to oval uniform nuclei, with inconspicuous to small nucleoli and without atypia. Duct profiles were surrounded by a thin rim of contractile fibromuscular stroma with normal cellularity and without atypia and more peripheral looser connective tissue. AR immunostaining showed the expected positive nuclear staining in both epithelium and surrounding stroma (Figure 5). Both epithelial and stromal compartments were consistently negative for Tag immunostaining (Figure 5)

Tissue recombinants composed of $12 \mathrm{~T}-7 \mathrm{f} \mathrm{BlE}$ and 12T-7f mUGM showed lobular expansion consisting of increased glandular spaces containing stratified markedly atypical epithelium with accompanying hypercellular stroma (Figure 5 and Supplementary Figure 2, Table 1). The histologic appearance was essentially identical to the DLP of intact $12 \mathrm{~T}-7 \mathrm{f}$ and other fast growing lines of LPB-Tag mice. ${ }^{9,16}$ In tissue recombinants the symmetry and uniform nature of the atypical epithelial cell lesion and the connection of small acinar spaces to central duct lumens with nuclear atypia were similar to the histopathologic phenotype of mPIN seen in intact LPB-Tag mice. ${ }^{16}$ The atypical epithelial cell component was similar to that noted in the 12T-7f BlE and rUGM recombinants, in which the stroma was normal (Figures 2, 3 and Supplementary Figure 1). The stromal hypercellularity in the $12 \mathrm{~T}-7 \mathrm{f} \mathrm{BlE}$ and 12T-7f mUGM recombinants was distinct from the stroma using wt mUGM (Supplementary Figure 2). The stromal hypercellularity was also diffuse and uniform, in general reminiscent of prostate stroma, but with increased cellularity, similar to that in intact $12 \mathrm{~T}-7 \mathrm{f}$ mice. $^{9,16}$ Also, consistent with the DLP of intact $12 \mathrm{~T}-7 \mathrm{f}$ mice, there was focally condensed, accentuated hypercellularity immediately subjacent to some gland spaces, where abundant mitotic cells were seen. Both AR and Tag immunostaining showed uniform nuclear immunoreactivity in both the atypical hypercellular epithelial cell and the hypercellular stroma compartment (Figure 5).

Tissue recombinants composed of $12 \mathrm{~T}-7 \mathrm{f} \mathrm{BlE}$ and wt mUGM showed lobular expansion of atypical epithelium, with stratification, nuclear enlargement and atypia (Figure 5 and Supplementary Figure 2, Table 1). However, the associated stroma was normal (Supplementary Figure 2). The overall appearance was similar to that in the 12T-7f BlE and rUGM recombinants (Figure 2), with a slightly thinner rim of contractile fibromuscular stroma generated with mUGM when compared to that with rUGM. AR immunostaining showed the expected uniform, diffuse nuclear immunoreactivity in both the epithelial cell and stromal compartment (Figure 5). Tag immunostaining showed uniform, diffuse and strong nuclear immunoreactivity in the proliferating

Figure $3 \mathrm{AR}$ and SV40T expression in 12T-7f plus rUGM recombinants. Immunoreactivity to antibodies against androgen receptor (AR; $\mathbf{a}, \mathbf{c}, \mathbf{e}$ and $\mathbf{g}$ ) and SV40 large T antigen (Tag; $\mathbf{b}, \mathbf{d}, \mathbf{f}$ and $\mathbf{h}$ ) in sections of tissue recombinants composed of 12T-7f BlE and rUGM harvested at 6 (a and $\mathbf{~ b ) , ~} 8$ (c and $\mathbf{d}$ ), 12 (e and f) and 34 (g and $\mathbf{h}$ ) weeks postgrafting. AR immunostaining is seen in both the proliferating atypical epithelium of the mPIN lesions, as well as in the surrounding stromal cells. In contrast, Tag immunostaining is seen only in the proliferating, atypical epithelial compartment. Note early induction of transgene expression, and also that AR and Tag are no longer uniform at 34 weeks, with focal-negative epithelial staining in an area noted as potentially representing a microscopic focus of invasion. 

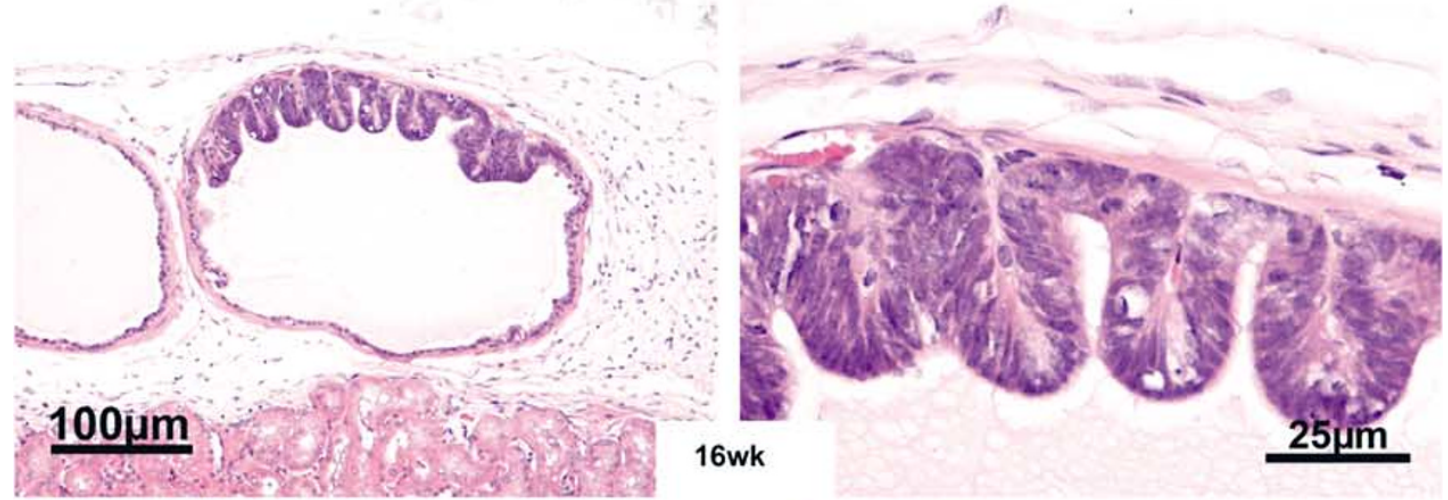

c

d
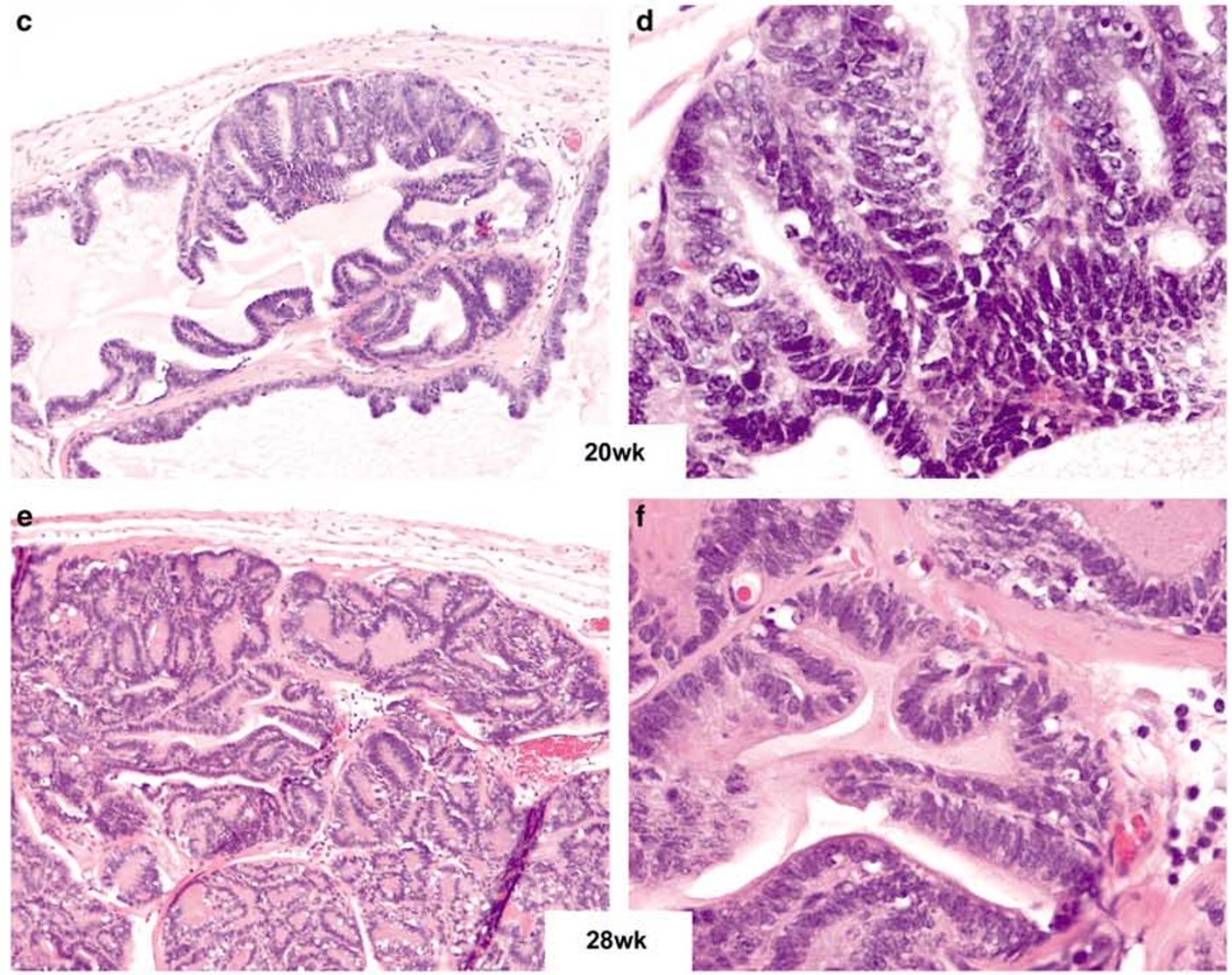

g
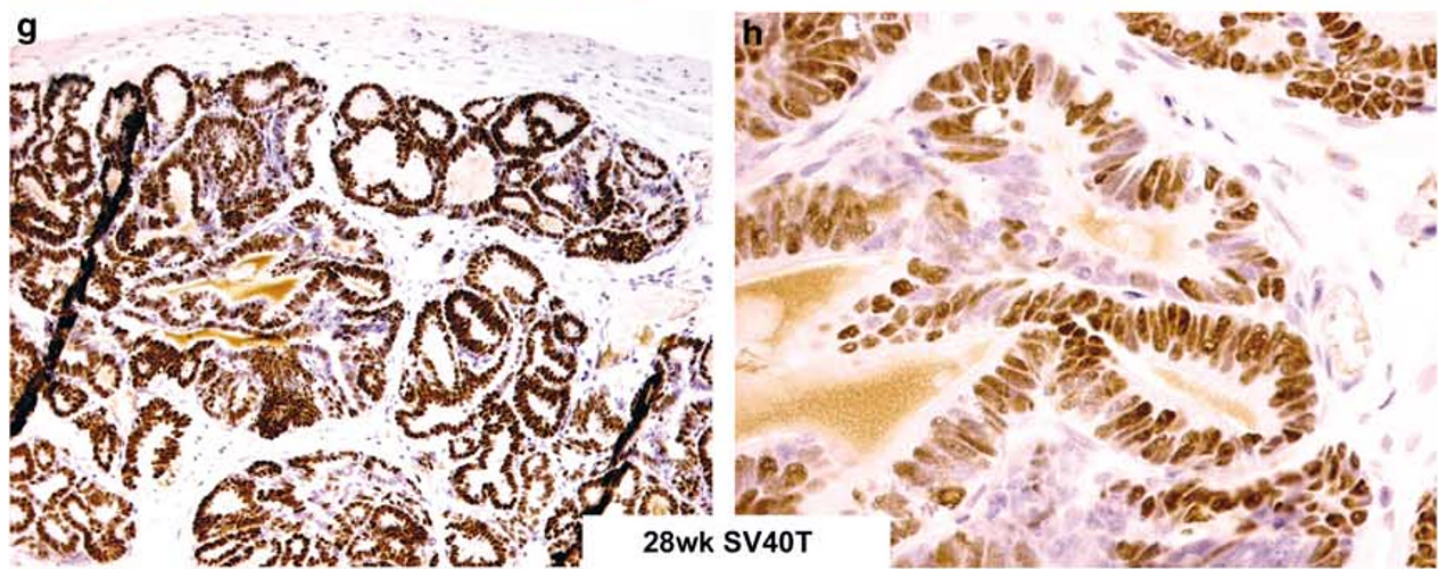
atypical epithelial component, and absence of Tag expression in the stromal compartment (Figure 5).

Tissue recombinants composed of wt BlE and 12T-7f mUGM showed a fairly normal epithelial compartment, similar to intact wt mouse prostate, surrounded by a markedly hypercellular stroma (Figure 5 and Supplementary Figure 2, Table 1). The stroma was similar in appearance to that of the hypercellular stroma in the DLP and AP of intact transgenic mice of the $12 \mathrm{~T}-7 \mathrm{f}$ and other fast growing LPB-Tag lines such as $12 \mathrm{~T}-7 \mathrm{~s}^{9,16,19}$ The stromal cells and surrounding eosinophilic collagenous matrix were reminiscent of the prostate fibromuscular stroma of normal mice, but cellularity was markedly increased, with readily apparent mitotic figures and mild nuclear atypia (Supplementary Figure 2). Also, similar to the stroma of DLP of intact $12 \mathrm{~T}-7 \mathrm{f}$ mice and recombinants made with $12 \mathrm{~T}-7 \mathrm{f} \mathrm{BlE}$ and $12 \mathrm{~T}-7 \mathrm{f}$ mUGM, immediately subjacent to the normalappearing epithelium, there was a surrounding rim of even more pronounced stromal cellularity. AR immunostaining showed expected nuclear staining of both the epithelial and stromal compartments (Figure 5). Tag immunostaining showed strong, diffuse nuclear immunoreactivity only in the hypercellular stroma (Figure 5). The normal-appearing prostate columnar epithelial compartment was Tag immunonegative, including foci histologically compatible with transitional metaplasia.

\section{Distribution of Tag Expression in Neoplastic Prostates of Intact LPB-Tag 12T-7f, 12T-7s, 12T-10 and 12T-11 and TRAMP (sPB-SV40) Mice}

Tag expression was noted in the hypercellular prostatic stroma of tissue recombinants made with mUGM from LPB-Tag 12T-7f mice. The histopathology of this stroma was similar to that noted in the intact prostates of fast growing LPB-Tag lines. Therefore, we further investigated possible unexpected transgene expression in four Tag lines made with the LPB promoter (12T-7f, 12T-7s, 12T-10 and 12T-11). ${ }^{17}$ The pattern of transgene expression in these mice as demonstrated by Tag immunostaining is summarized in Tables 2 and 3 and examples illustrated in Figure 6 and Supplementary Figure 3. Of note, intense immunopositive reactions for the Tag transgene expression were seen in the neoplastic prostatic epithelium of all four lines. In three lines (12T-7f, 12T-7s and 12T-11), Tag immunostaining was also observed in the prostatic stroma.
A number of nonprostatic sites were also found to be Tag immunoreactive, including the stroma of the seminal vesicle (which demonstrates varying degrees of hypercellularity between the transgenic mouse lines). ${ }^{16}$ In the $12 \mathrm{~T}$-7f mouse the seminal vesicle wall exhibited a hypercellular stroma that generally resembled the normal contractile seminal vesicle smooth muscle, with typically mild atypia and progressively increased cellularity. However, focally distinct nodule-like areas of more accentuated increased stromal cellularity, with greater atypia and mitoses were occasionally noted (discussed in Shappell et $a l^{16}$ ), now raising consideration of Tag-induced stromal sarcomas/ leiomyosarcomas.

Strong nuclear Tag immunostaining was also seen in focal areas of hypercellular and mildly atypical keratinocyte or follicular epithelium, adjacent to cutaneous adnexa was seen in the ears of $12 \mathrm{~T}-7 \mathrm{f}$ mice. However, similar histopathologic alterations and associated Tag immunostaining were not seen in representative areas of epidermis and cutaneous adnexa in the skin of 12T-10 mice. Epididymal stromal Tag expression was particularly interesting, with different segment-specific variations of positive stromal and epithelial immunostaining in each line (summarized in Table 3 and illustrated in Supplementary Figure 3). Although immunohistochemical analysis is not strictly quantitative it is notable that in all mice examined Tag immunoreactivity was consistently most intense in the prostatic epithelium compared to other cell types. In some stromal locations, notably in parts of the epididymis, Tag staining intensity approached that of the stroma in the prostate but was less intense than the prostatic epithelium. In epididymal epithelium, Tag immunostaining, while unequivocally positive when compared to wt, was much less intense than prostatic epithelium and the epididymal nuclei did not exhibit the elongated phenotypeassociated dysplastic cells.

We similarly examined Tag expression in the less pronounced, but well-described, hypercellular stroma observed in the prostate lesions of the TRAMP model. ${ }^{16}$ The results, summarized in Figure 7 , demonstrate that Tag expression in the TRAMP prostatic stroma closely resembled that seen in the LPB-Tag 12T-7f model. Tag immunostaining was noted in the neoplastic epithelium of TRAMP accompanied by extensive positive Tag immunostaining in the nuclei of the adjacent hypercellular stroma (Figure 7).

Figure 4 Phenotype of tissue recombinants composed of 12T-10 BlE and rUGM. Intermediate- and high-power photomicrographs, respectively, of H\&E-stained sections of recombinants harvested at 16 (a and b), 20 (c and d) and 28 (e and f) weeks postgrafting. Temporally progressive epithelial proliferations, in terms of both extent and degree of nuclear atypia, compatible with mPIN in intact mouse prostate, are evident. Particularly at the 16 and 20 weeks time points, the slower development compared to similar recombinants with 12T-7f epithelium (Figure 1), is evident. The less pronounced lobular expansion of the mPIN lesion with the 12T-10 epithelium, with atypical epithelium more confined to ducts with more normal architecture (a and $\mathbf{c}$ ) is also similar to the phenotypic differences in intact 12T-10 vs 12T-7 mouse DLPs. (g and h) Intermediate- and high-power photomicrographs of Tag-immunostained sections at 28 weeks postgrafting, showing expression in neoplastic epithelium, but not surrounding normal stroma. 
Table 1 Tissue recombinations and resulting histology used to investigate the role of stromal and epithelial transgene expression in the genesis of stromal hypercellularity in LPB-Tag mice

\begin{tabular}{lllll}
\hline \multicolumn{2}{c}{ Tissue recombinants } & & \multicolumn{2}{c}{ Result } \\
\cline { 1 - 1 } Epithelium & Stroma & & Epithelium & Stroma \\
\hline $\mathrm{Wt}$ & $\mathrm{Wt}$ & & Normal & Normal \\
$\mathrm{Tag}$ & $\mathrm{Tag}$ & & mPIN & Hypercellular \\
$\mathrm{Tag}$ & $\mathrm{Wt}$ & & mPIN & Normal \\
$\mathrm{Wt}$ & $\mathrm{Tag}$ & & Normal & Hypercellular \\
\hline
\end{tabular}

Another notable finding was the extensive positive Tag immunostaining in both the hypercellular stromal and epithelial compartments of the combined epithelial and stromal lesions ${ }^{16}$ previously designated as 'phyllodes-like' in TRAMP prostates $^{29}$ (Figure 7). Although the hypercellular and possibly neoplastic stromal component of such lesions has been recognized, it has not previously been appreciated that these foci correlate with SV40T-antigen transgene expression in the TRAMP model. Additional findings in the TRAMP model included the observation of occasional transgene expression in the stromal but not the epithelial cells of the seminal vesicle (Figure 7) and strong positive Tag immunostaining in areas of atypical epithelial hyperplasia ${ }^{16}$ of the periurethral glands (Figure 7), similar to that previously noted in the C3(1)-SV40 mouse ${ }^{30}$ and the LPB-Tag 12T-10 mouse. ${ }^{16,17,19,21}$

\section{Discussion}

The present study was designed to determine whether a tissue recombination model would accurately recapitulate disease progression in wellcharacterized transgenic models of prostate cancer. This is an important step in validating the use of tissue recombination as a screening tool for pretesting genes that later will be used to create new transgenic animal models. The results demonstrated a close correlation between the epithelial phenotypes of intact transgenic mice and tissue recombi- nation models. This study used both fetal UGE and adult bladder epithelium as starting materials in tissue recombinants. The results obtained were essentially identical with either starting material. However, the bulk of the data described here was from tissue recombinants prepared using bladder epithelium. Bladder epithelium is readily available, technically simple to prepare and allows controlled temporal exposure of cells to the Tag transgene. Tag is not expressed in the bladder urothelium of $12 \mathrm{~T}-7 \mathrm{f}$ or 12T-10 mice; however, expression of the transgene is initiated rapidly upon tissue recombination with UGM.

Tissue recombinants composed of $12 \mathrm{~T}-7 \mathrm{f} \mathrm{BlE}$ and wt rat or mouse UGM gave rise to PIN lesions consistent with those seen in the prostate of the 12T$7 \mathrm{f}$ transgenic line. These recombinants mimic the in vivo postpubertal progression of the disease with the rapid induction of Tag expression and the development of PIN lesions. In contrast, when the epithelium from 12T-10 mouse bladder was used in the recombinants, progression was slow and the tissue recombinant mirrored the slowly developing tumor of the 12T-10 transgenic line. These findings demonstrate that the tissue recombination model reflects not only the overall organizational events but also temporal details of tumor progression in a transgenic model. Thus, tissue recombination is a good system to pretest the consequences that altering a gene has on prostatic phenotypic before targeting disruption of that gene in transgenic mice.

In order to pretest genes for models of prostate cancer, we would propose using wt rUGM recombined with mouse prostatic epithelial cells where candidate genes are introduced or suppressed using viral vectors (see Hayward ${ }^{31}$ for further discussion of possible methods). Tissue recombination has been used in many different organs, notably the mammary gland and gastrointestinal tract suggesting that such screening methods could be used in applications beyond prostate cancer.

The histology of the $12 \mathrm{~T}-7 \mathrm{f}$ prostate includes marked nuclear atypia, with enlarged hyperchromatic nuclei and abundant mitotic figures. There is a clear increase in gland spaces compared to agematched wt mice. However, this proliferative lesion is symmetric and uniform. The small acinar spaces

\footnotetext{
Figure 5 Defining the tissue specificity of SV40T expression in 12T-7f tissue recombinants. Androgen receptor (AR; a, c, e and g) and SV40 large T-antigen (Tag; $\mathbf{b}, \mathbf{d}, \mathbf{f}$ and $\mathbf{h}$ ) immunostaining in sections of tissue recombinants composed of wt BlE and wt mUGM (a and $\mathbf{b}$ ), 12T-7f BlE and 12T-7f mUGM (c and d), 12T-7f BlE and wt mUGM (e and f), and wt BlE and 12T-7f mUGM (g and h). (a and b) Uniform nuclear AR immunostaining (a) and absence of Tag expression (b) in both normal-appearing epithelium and surrounding normal cellular stroma in recombinants of wt mouse epithelium and wt mUGM. (c and d) Recombinants made with both epithelium and mUGM from 12T-7f show AR immunostaining is in both the neoplastic epithelial compartment and the hypercellular stroma (c) while Tag immunostaining demonstrates uniform nuclear immunoreactivity in both the atypical epithelial compartment and the hypercellular stroma (d). (e and f) AR immunostaining in both atypical proliferating epithelium of mPIN lesion and surrounding normocellular stroma, in contrast to presence of Tag in neoplastic epithelium but wt stroma (f) of recombinants of 12T-7f epithelium and wt mUGM. (g and h) AR immunostaining in nuclei of epithelium of normal appearing prostate duct/gland (with absence of stratification or atypia) and in nuclei of markedly hypercellular and mildly atypical surrounding stroma (g); absence of Tag immunostaining in normal epithelium but strong, essentially uniform Tag immunostaining in hypercellular stroma (h) of recombinants made with wt mouse epithelium and LPBTag 12T-7f UGM. Note that in contrast to absence of Tag in normal stroma of recombinants made with rUGM and wt mUGM, stromal Tag expression is associated with stromal hypercellularity and requires the use of 12T-7f UGM.
} 

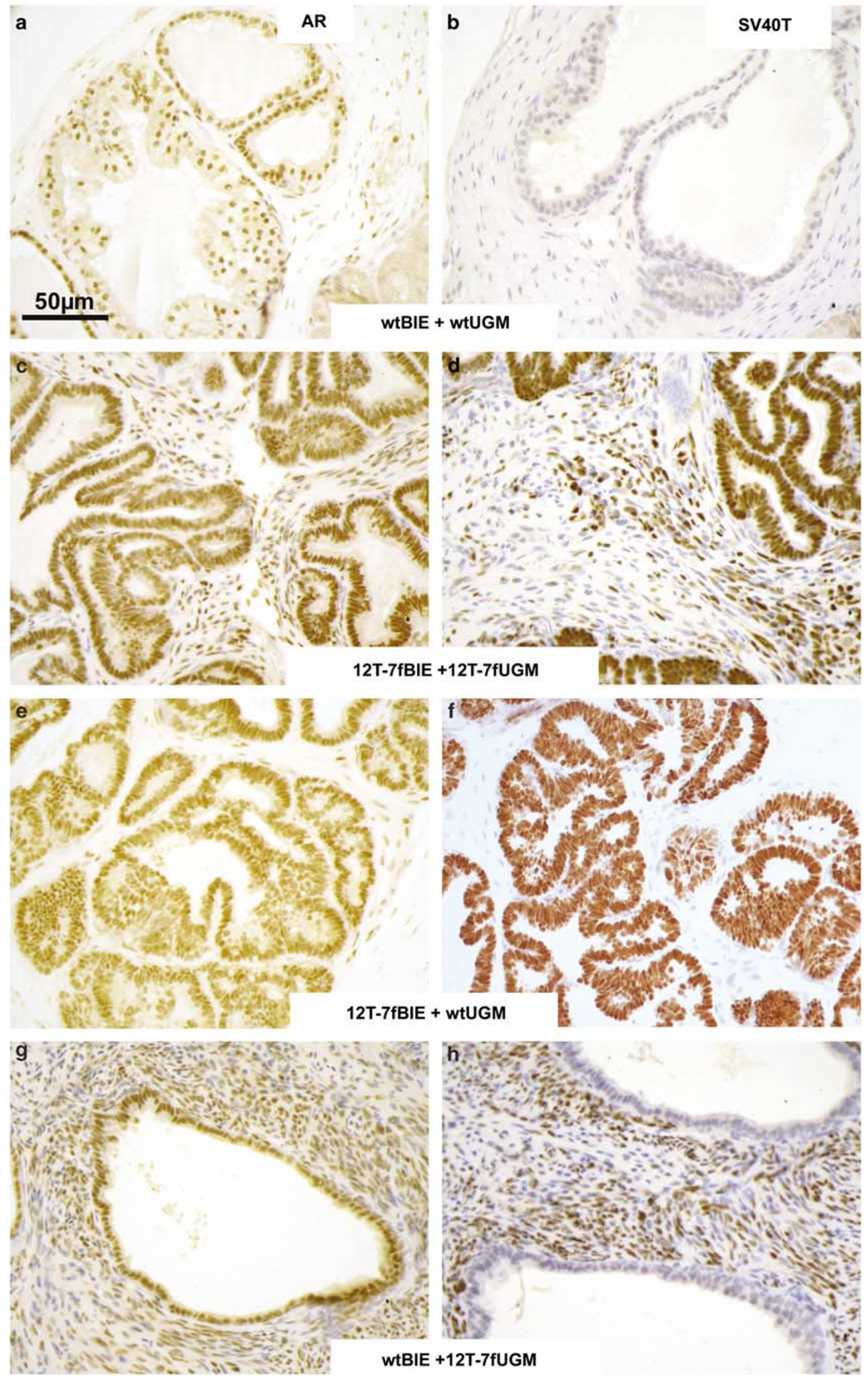
Table 2 Localization of expression of Tag in a variety of tissues in four LPB-Tag mouse strains

\begin{tabular}{|c|c|c|c|c|}
\hline Organ & $12 T-7 f$ & $12 \mathrm{~T}-7 \mathrm{~s}$ & $12 T-10$ & $12 T-11$ \\
\hline Prostate & Epi and Stroma & Epi and Stroma & Epi & Epi and Stroma \\
\hline Seminal vesicle & Stroma & Stroma & Epi & Stroma \\
\hline Ductus deferens & Stroma & Absent & Absent & Absent \\
\hline Testis & Absent & Absent & Absent & Absent \\
\hline Kidney & Absent & Absent & Absent & Absent \\
\hline Bladder & Absent & Absent & Absent & Absent \\
\hline Intestine & Absent & Absent & Absent & Absent \\
\hline Stomach & Absent & Absent & Absent & Absent \\
\hline Liver & Absent & Absent & Absent & Absent \\
\hline Spleen & Absent & Absent & Absent & Absent \\
\hline Lung & Absent & Absent & Absent & Absent \\
\hline Heart & Absent & Absent & Absent & Absent \\
\hline Skeletal muscle & Absent & Absent & Absent & Absent \\
\hline Skin (Ear) & Sporadic Epi & Sporadic Epi & Absent & Absent \\
\hline Brain & Absent & Absent & Absent & Absent \\
\hline
\end{tabular}

Staining was consistently the most intense in the epithelium (Epi) of the prostate but was also observed with variable intensity in both stromal and epithelial cells in a number of organs.

Table 3 Expression of Tag along the epididymis of four strains of LPB-Tag mice

\begin{tabular}{|c|c|c|c|c|c|}
\hline & \# & $12 T-7 f$ & $12 T-7 s$ & $12 T-10$ & $12 T-11$ \\
\hline Efferent duct (EF) & & Stroma & Stroma & Negative & Missing \\
\hline Initial segment (IS) & 1 & Stroma & Stroma & Stroma & Stroma \\
\hline \multicolumn{6}{|l|}{ Caput epididymis } \\
\hline Proximal caput epididymis & 1 & Stroma & Stroma & Stroma & Stroma \\
\hline Proximal caput epididymis & 2 & Stroma & Stroma & Stroma & Stroma \\
\hline Distal caput epididymis & 3 & Stroma & Stroma & Epi & Stroma \\
\hline Distal caput epididymis & 4 & Stroma & Stroma & Epi & Stroma \\
\hline Distal caput epididymis & 5 & Stroma & Stroma & Epi & Stroma \\
\hline Corpus epididymis & 6 & Epi & Epi & Epi & Epi \\
\hline \multicolumn{6}{|l|}{ Cauda epididymis } \\
\hline Proximal cauda epididymis & 7 & Epi & Epi & Epi & Epi \\
\hline Distal cauda epididymis & 8 & Stroma & Negative & Negative & Negative \\
\hline Vas deferens & & Muscle & Negative & Negative & Negative \\
\hline
\end{tabular}

Note that the staining level is highly variable (see Supplementary Figure 3 for visual comparison). For example the epithelial staining was of much lower intensity than the stromal staining. \# is epididymal segment as described in Abou-Haila and Fain-Maurel. ${ }^{32}$

at the periphery of the lobules appear to connect up to the central dilated duct lumens with similar nuclear atypia and cytologic character in both. This appearance has led to the interpretation of this lesion as an in situ neoplastic process. ${ }^{16}$ Since this lesion progresses in time, it is regarded according to standardized criteria, as mPIN. ${ }^{16}$ Increased cellularity of the surrounding fibromuscular stroma is evident with atypical nuclei and Tag expression seen in the hypercellular stroma.

Figure 6 SV40T expression in LPB-Tag mice. SV40 large T-antigen (Tag) immunostaining in the neoplastic prostate (a and b), seminal vesicle ( $\mathbf{c}$ and $\mathbf{d}$ ), epididymis (e and $\mathbf{f})$ and ear skin ( $\mathbf{g}$ and $\mathbf{h})$ in sections from intact adult LPB-Tag $12 \mathrm{~T}-7 \mathrm{f}(\mathbf{a}, \mathbf{c}, \mathbf{e}$ and $\mathbf{g})$ and $12 \mathrm{~T}-10$ (b, $\mathbf{d}, \mathbf{f}$ and h) mice. (a) Strong uniform Tag expression in neoplastic epithelium of mPIN lesion and the associated hypercellular stroma of DLP of 12T-7f mouse. (b) Tag expression in atypical nuclei of epithelium of DLP of 12T-10 mouse, but absence of Tag expression in essentially normocellular stroma. Note typical less dramatic expansion of atypical epithelial compartment in slow growing $12 \mathrm{~T}-10 \mathrm{line}$ vs $12 \mathrm{~T}-7 \mathrm{f}$ shown in a, as well as profound difference in stroma phenotype and Tag expression between the lines. (c) Uniform Tag expression in hypercellular stroma, but absence of Tag expression in normal appearing epithelium, of seminal vesicle in $12 \mathrm{~T}-7 \mathrm{f}$ mouse. (d) Absence of Tag immunostaining in both normal appearing seminal vesicle epithelium and stroma (bottom right) of similar section from 12T-10 mouse. (e) Presence of Tag expression in stroma and absence of expression in normal-appearing epithelial compartment of epididymis in $12 \mathrm{~T}-7 \mathrm{f}$ mouse. (f) Absence of Tag expression in both normal-appearing epithelium and associated stroma in epididymis in 12T-10 mouse. Note uniform expression in prostatic epithelium in both strains with stromal staining in the SV stroma, epididymal stroma and skin restricted to the 12T-7 f line and not seen in the 12T-10 line. (g) Strong nuclear Tag immunostaining in focal area of hypercellular and mildly atypical keratinocyte or follicular epithelium (arrowhead), adjacent to cutaneous adnexa (top left). (h) Absence of similar histopathologic alteration and Tag immunostaining in representative area of epidermis and cutaneous adnexa (center) in skin of 12T-10 mouse. 


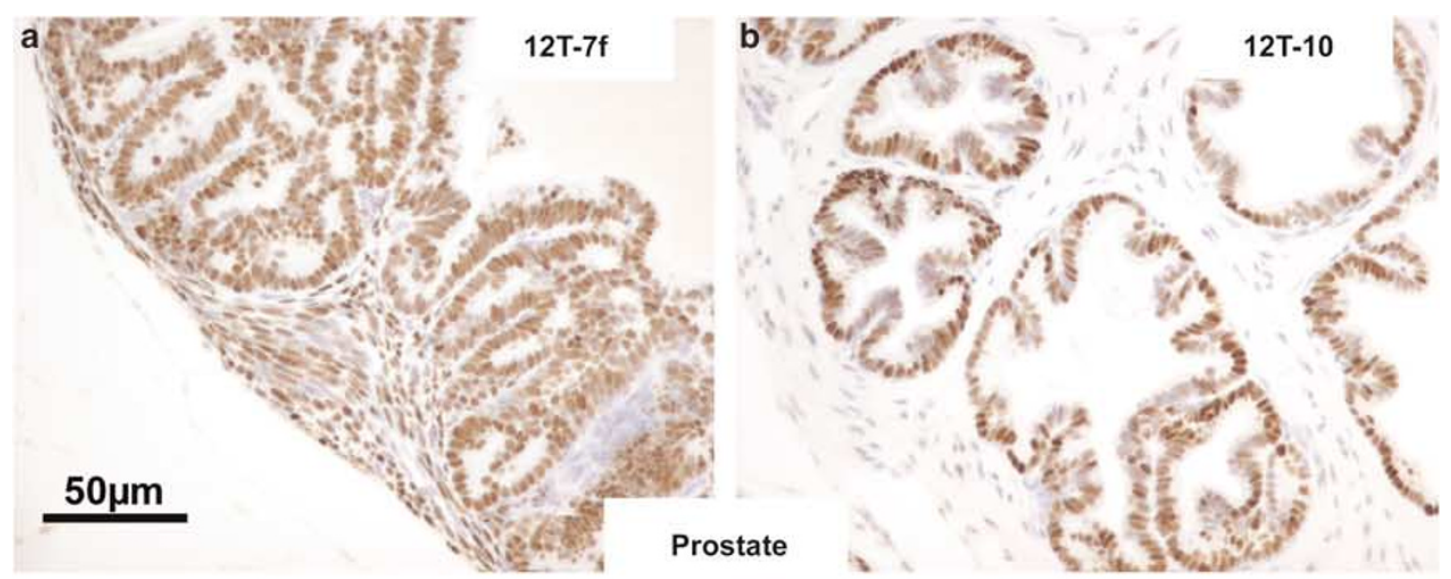

c
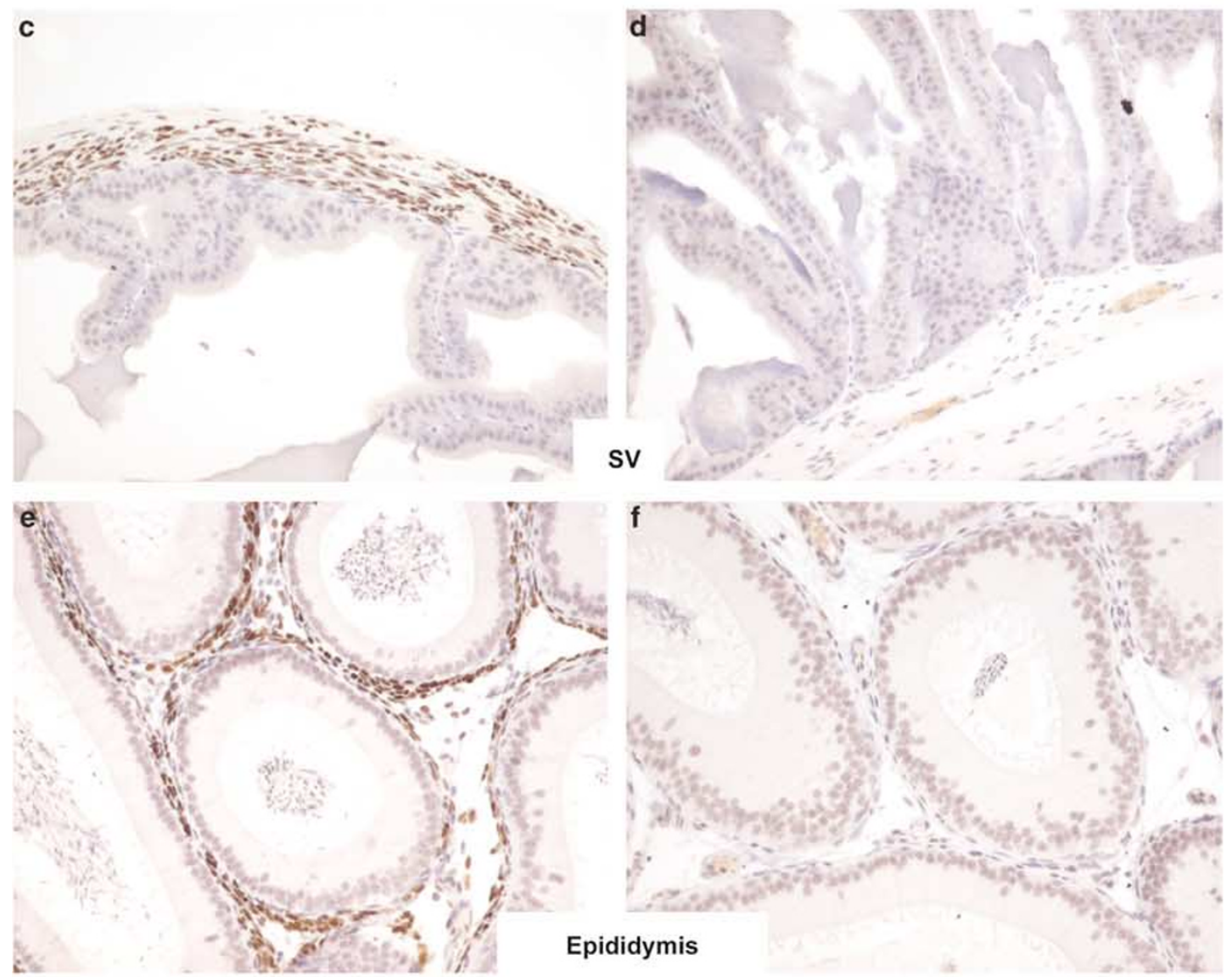

g

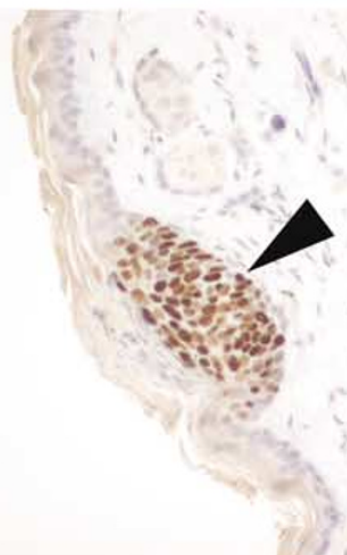

h

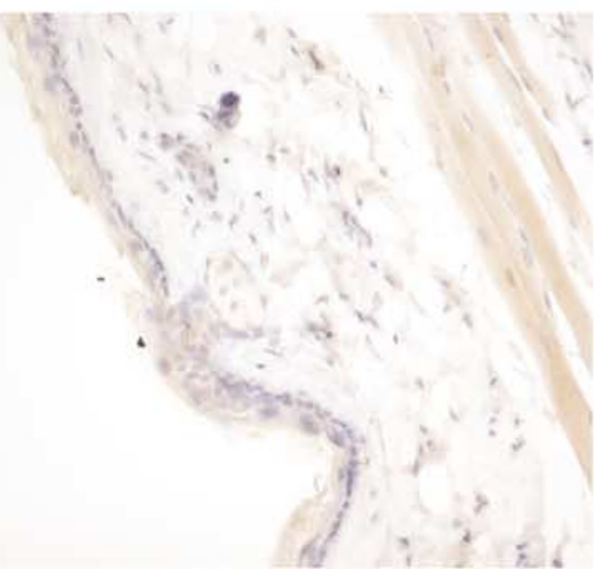

Ear 
1100
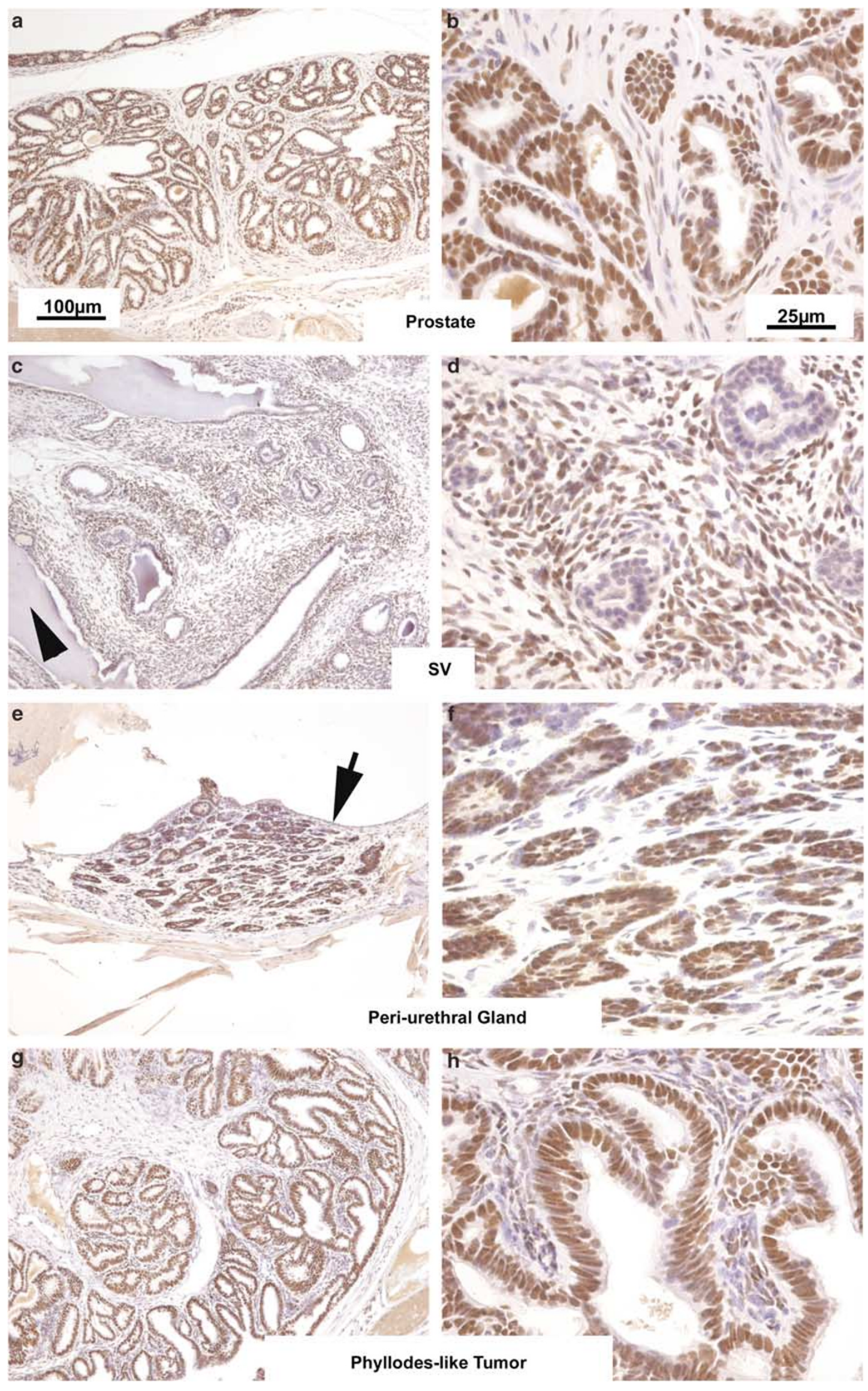
The observation that tissue recombinants made with wt UGM exhibited a close to normal stromal morphology as compared to the Tag-expressing hypercellular stroma prompted us to revisit this phenomenon. There are two obvious possible explanations for such an observation. First, stromal hypercellularity can result from epithelial to mesenchymal transformations (EMTs) occurring within the established tumor, allowing epithelial cells expressing Tag to appear as stroma. A second option is that Tag is expressed in the fetal mesenchymederived stromal cells. It is noted that these two options are not mutually exclusive. To address this issue we performed a series of tissue recombinants, which conclusively demonstrated that hypercellular stroma can result from Tag expression in the mesenchyme-derived stroma and does not require EMTs. We found no evidence for the occurrence of EMTs in the TR model but cannot totally exclude the possibility of EMT occurrence in the transgenic lines.

An exhaustive examination of Tag expression in 15 organs of four LADY lines yielded data demonstrating a localized Tag expression in the skin (ear) for two lines, ductus deferens stroma for one line, and seminal vesicle expression in the stroma of three lines and the epithelium of one line (Table 2). The epididymis is divided into specific segments ${ }^{32}$ that show region-specific expression of six lipocalins. ${ }^{7}$ Surprisingly, LPB-Tag expression shows distinct stroma and/or epithelial expression in an epididymal-segment-specific manner (Table 3). This suggests that the epididymal lipocalin gene cluster on chromosome 2 and the PB-related lipocalin cluster on the X-chromosome share similar regulatory sequences.

By comparison to other organs, Tag expression in the LADY models was greatest in the prostatic epithelium. However, there were clear and marked differences in the expression pattern among the four LADY lines. Three LADY lines showed Tag expression in the prostatic epithelial and stroma cells while Tag was detected only in the epithelium of the
12T-10 line (Table 2). Expression of Tag in the stroma was surprising since the $\mathrm{PB}$ promoter routinely targets the prostatic epithelium. However, with the exception of skin in the ear, all observed stromal Tag expression was confined to male reproductive organs that are androgen regulated. It is reasonable to suggest that the stroma and epithelium of the male reproductive organs share, at least in part, a common mechanism that involves the androgen receptor to dictate gene expression in a cell-specific manner. Further, these data underline the idea that transgene insertion has a clear influence on the final pattern of gene expression even if the promoter is tissue specific.

An investigation of TRAMP urogenital tract tissues likewise demonstrated inappropriate expression of the Tag transgene under the control of the small PB promoter. As expected Tag expression was seen throughout the prostatic epithelium of the TRAMP samples investigated. Staining was also noted in the prostatic stroma. We demonstrated that the 'phyllodes-like' tumors reported in the TRAMP model $^{20}$ exhibit stromal Tag expression that is consistent with descriptions of the sarcomatous nature of the phyllodes tumors after which these lesions are named. Such lesions have been classified in the NCI MMHCC scheme ${ }^{16}$ as combined epithelial and stromal hyperplasias, with atypia in the epithelial and potentially in the stromal compartment. This characterization recognizes the potential neoplastic nature as well as the utility of the descriptive modifier 'phyllodes-like'. Consistent with the data presented here it has recently been suggested that 'phyllodes-like' TRAMP lesions are likely the result of transgene expression in the stroma leading to stromal hyperproliferation and mixed epithelial-stromal tumors. ${ }^{33}$

In addition to the stromal expression of Tag in the TRAMP prostate, occasional transgene expression was seen in the stromal but not the epithelial cells of the seminal vesicle reminiscent of the positive Tag immunostaining in focal areas of seminal vesicle hypercellular stroma in LPB-Tag $12 \mathrm{~T}-7 \mathrm{f}$ mice. The

Figure 7 SV40 T-antigen expression in TRAMP mouse tissues. SV40 large T-antigen (Tag) expression in epithelium and stroma of various neoplastic lesions in androgen-regulated male accessory glands in TRAMP model. (a and b) Positive Tag immunostaining in neoplastic epithelium and accompanying hypercellular stroma in lesions regarded as mPIN by the NCI MMHCC criteria. (a) Note uniform Tag immunostaining in all areas of neoplastic epithelium. (b) Higher power showing uniform strong nuclear Tag immunostaining in the atypical epithelial compartment as well as extensive positive nuclear Tag immunostaining in mildly atypical hypercellular stroma between immunopositive glands. (c and $\mathbf{d}$ ) Positive Tag immunostaining in mildly atypical hypercellular stroma and absence of Tag immunostaining in essentially normal-appearing epithelium of seminal vesicle from same TRAMP mouse as in (a and b). (c) A polypoid like protrusion of seminal vesicle epithelium and stroma into seminal vesicle lumen (arrowhead). Tag immunopositive hypercellular stroma is seen surrounding more normal-appearing epithelial profiles in a collar-like fashion. (d) At higher power, diffusely positive Tag nuclear immunostaining is seen in the mildly atypical, prominently hypercellular stroma surrounding epithelium as well as in the less cellular foci of stroma further away from the epithelium. In contrast, the histologically and cytologically normal seminal vesicle epithelium is Tag immunonegative. (e and f) Strong, uniform Tag immunostaining in focus of atypical hyperplasia in periurethral glands in TRAMP mouse. Histologically, there is epithelial stratification and nuclear atypia, including enlargement and prominent hyperchromasia, without frank invasion into surrounding stroma. Tag immunostaining was not noted in overlying, histologically normal urothelium (arrow). (g and h) Intermediate- and high-power photomicrograph showing Tag immunostaining in atypical epithelium as well as hypercellular stroma of a lesion termed phyllodes-like by the developers of TRAMP. Such lesions commonly protrude into the prostate duct lumens and are also seen in older LPB-Tag 12T- $7 \mathrm{f}$ mice. (h) At higher magnification, the diffuse expression of Tag in the stratified, atypical epithelium is shown. Extensive nuclear Tag immunostaining is also noted in the atypical hypercellular stroma surrounding the epithelial component. 
epididymis of TRAMP was not examined. Tag staining was however seen in the periurethral gland epithelium of TRAMP. The periurethral glands are androgen-regulated male accessory glands. Lesions seen in these have been classified as atypical epithelial hyperplasia in the NCI MMHHC ${ }^{16}$ classification scheme. In the C3(1)-SV40 mouse and LPBTag mouse, such lesions have been seen prior to or at the same time as invasive poorly differentiated carcinomas, including tumors with NE differentiation. These lesions are similar to the unequivocal invasive Pcas and metastatic carcinomas in these models as well as TRAMP. The expression of Tag in these other male accessory tissues with the sPB promoter in TRAMP is hence similar to that previously reported with the C3(1) and LPB promoters.

The key characteristic of both the TRAMP and LADY models is their ability to generate prostate tumors. In both of these models, transgene expression is most uniform and intense in the prostatic epithelium. In other sites the transgene was expressed weakly and often sporadically. Thus, the prostate epithelium is the tissue that receives the most concentrated dose of the transgene, presumably explaining why the bulk of the tumors seen in these animals are epithelial in orgin. There is considerable anecdotal evidence from many groups using these models, of nonprostate tumors cropping up sporadically. However, there has been no comprehensive report on these phenomena and scant formal documentation of the incidence of these tumors.

The similarities and differences between the various PB promoter-driven models described here and elsewhere parallel in some ways those recently reported in two $\mathrm{ARR}_{2} \mathrm{~PB}$-Cre lines. ${ }^{34}$ Thus all three versions of the $\mathrm{PB}$ promoter $\left(\mathrm{sPB},^{8} \mathrm{LPB}^{2,9}\right.$ and $\mathrm{ARR}_{2} \mathrm{~PB}^{3}$ ) exhibit somewhat more varied expression patterns than had previously been appreciated. In part, imprinting on the PB promoter may occur since differences in the pattern of gene expression are reported based upon the transgene being passed from female or male mice breeders. ${ }^{34}$ Further, the specific distribution pattern seems to be influenced by the site of integration. This strongly suggests that transgene insertion plays an important role in the fine tuning of PB-driven transgenes in their ability to target prostatic epithelium. A complex group of transcription factors, shared by many tissues, interact to regulate expression of prostate-specific genes. $^{35}$ It seems that while there is a baseline phenotype typically generated by introducing a promoter-transgene construct, it is likely that there will be variations upon this theme that depend upon the specific integration site. This phenomenon is unlikely to be limited to the use of the PB promoter but may well occur in any transgenic model. Indeed the fact that such a well-characterized promoter gives rise to these effects only emphasizes the need for care in the interpretation of data derived from transgenic mice. An important consequence of these observations is to reinforce the idea that detection of transgene expression at a site distant from a primary tumor in a transgenic model is not, of itself, a marker of metastasis. Linkage to the primary tumor is clearly necessary to define metastatic sites. ${ }^{21}$

In conclusion, successful tissue reprogramming of adult 12T-7f and 12T-10 LPB-Tag bladder epithelium to prostatic epithelium was achieved, resulting in tissue recombination progression, which mirrored the autochthonous disease in the transgenic models. Further, these studies demonstrate that tissue recombination approaches can be used to dissect the contributions of stromal and epithelial cell populations to the overall development of the tumor in transgenic models. This is an important proof-ofprinciple for the use of tissue recombinants as a phenotypic screening tool to pretest genes that will later be used in the generation of new transgenic models of disease.

\section{Acknowledgements}

This work was supported by NCI Grant U01CA96403 and funding from the Joe C Davis Foundation to SWH and by R01-CA76142 and the Frances Williams Preston Laboratories of the TJ Martell Foundation to RJM. We are indebted to Marie-Claire Orgebin-Crist and Kichiya Suzuki for sharing their expertise on epididymal morphology and to Karin Williams, Tom Case, Romano DeMarco, Suzanne Fernandez and Harold Love for their assistance.

\section{References}

1 Greenberg N, DeMayo F, Sheppard P, et al. The rat probasin gene promoter directs hormonally and developmentally regulated expression of a heterologous gene specifically to the prostate in transgenic mice. Mol Endocrinol 1994;8:230-239.

2 Yan Y, Sheppard PC, Kasper S, et al. Large fragment of the probasin promoter targets high levels of transgene expression to the prostate of transgenic mice. Prostate 1997;32:129-139.

3 Zhang J, Thomas TZ, Kasper S, et al. A small composite probasin promoter confers high levels of prostate- specific gene expression through regulation by androgens and glucocorticoids in vitro and in vivo. Endocrinology 2000;141:4698-4710.

4 Spence A, Sheppard P, Davie J, et al. Regulation of a bifunctional mRNA results in synthesis of secreted and nuclear probasin. Proc Natl Acad Sci USA 1989;86: 7843-7847.

5 Johnson MA, Hernandez I, Wei Y, et al. Isolation and characterization of mouse probasin: an androgenregulated protein specifically expressed in the differentiated prostate. Prostate 2000;43:255-262.

6 Matusik RJ, Kreis C, McNicol P, et al. Regulation of prostatic genes: role of androgens and zinc in gene expression. Biochem Cell Biol 1986;64:601-607.

7 Suzuki K, Lareyre JJ, Sanchez D, et al. Molecular evolution of epididymal lipocalin genes localized on the mouse chromosome 2. Gene 2004;339:49-59. 
8 Greenberg NM, DeMayo F, Finegold MJ, et al. Prostate cancer in a transgenic mouse. Proc Natl Acad Sci USA 1995;92:3439-3443.

9 Kasper S, Sheppard PC, Yan Y, et al. Development, progression, and androgen-dependence of prostate tumors in probasin-large $\mathrm{T}$ antigen transgenic mice: a model for prostate cancer. Lab Invest 1998;78:319-333.

$10 \mathrm{Wu} \mathrm{X}, \mathrm{Wu} J$, Huang J, et al. Generation of a prostate epithelial cell-specific Cre transgenic mouse model for tissue-specific gene ablation. Mech Dev 2001;101: 61-69.

11 Lowe SL, Rubinchik S, Honda T, et al. Prostate-specific expression of Bax delivered by an adenoviral vector induces apoptosis in $\mathrm{LNCaP}$ prostate cancer cells. Gene Therapy 2001;8:1363-1371.

12 Andriani F, Nan B, Yu J, et al. Use of the probasin promoter ARR2PB to express Bax in androgen receptor-positive prostate cancer cells. J Natl Cancer Inst 2001;93:1314-1324.

13 Wen Y, Giri D, Yan DH, et al. Prostate-specific antitumor activity by probasin promoter-directed p202 expression. Mol Carcinog 2003;37:130-137.

14 Song Z, Wu X, Powell WC, et al. Fibroblast growth factor 8 isoform $B$ overexpression in prostate epithelium: a new mouse model for prostatic intraepithelial neoplasia. Cancer Res 2002;62:5096-5105.

15 Ellwood-Yen K, Graeber TG, Wongvipat J, et al. Mycdriven murine prostate cancer shares molecular features with human prostate tumors. Cancer Cell 2003;4: 223-238.

16 Shappell SB, Thomas GV, Roberts RL, et al. Prostate pathology of genetically engineered mice: definitions and classification. The consensus report from the Bar Harbor meeting of the Mouse Models of Human Cancer Consortium Prostate Pathology Committee. Cancer Res 2004;64:2270-2305.

17 Kasper S, Tu W, Roberts RL, et al. Transgenic mouse models for prostate cancer. Identification of an androgen-dependent promoter and creation and characterization of the long probasin promoter-large $\mathrm{T}$ antigen (LPB-Tag) model. Methods Mol Med 2003;81:113-147.

18 Matusik RJ, Masumori N, Thomas T, et al. Transgenic mouse models of prostate cancer. In: Matzuk M, Brown CW, Kumar TR (eds). Contemporary Endocrinology: Transgenics in Endocrinology. Humana Press Inc.: Totowa, NJ, 2001, pp 401-425.

19 Shappell SB, Masumori N, Thomas T, et al. Transgenic mouse models of prostate carcinoma: anatomic, histopthologic and molecular considerations. In: Abel PD, Lalani E-N (eds). Prostate Cancer Clinical and Scientific Aspects-Bridging the Gap. Imperial College Press: London, 2003, pp 245-320.

20 Evangelou AI, Winter SF, Huss WJ, et al. Steroid hormones, polypeptide growth factors, hormone refractory prostate cancer, and the neuroendocrine phenotype. J Cell Biochem 2004;91:671-683.

21 Masumori N, Thomas TZ, Chaurand $\mathrm{P}$, et al. A probasin-large $\mathrm{T}$ antigen transgenic mouse line develops prostate adenocarcinoma and neuroendocrine carcinoma with metastatic potential. Cancer Res 2001; 61:2239-2249.
22 Klezovitch O, Chevillet J, Mirosevich J, et al. Hepsin promotes prostate cancer progression and metastasis. Cancer Cell 2004;6:185-195.

23 Neubauer BL, Chung LWK, McCormick KA, et al. Epithelial-mesenchymal interactions in prostatic development. II. Biochemical observations of prostatic induction by urogenital sinus mesenchyme in epithelium of the adult rodent urinary bladder. J Cell Biol 1983;96:1671-1676.

24 Cunha GR, Fujii H, Neubauer BL, et al. Epithelialmesenchymal interactions in prostatic development. I. Morphological observations of prostatic induction by urogenital sinus mesenchyme in epithelium of the adult rodent urinary bladder. J Cell Biol 1983;96: 1662-1670.

25 Cunha GR, Donjacour AA. Mesenchymal-epithelial interactions: technical considerations. In: Coffey DS, Bruchovsky N, Gardner WA, Resnick MI, Karr JP (eds). Assessment of Current Concepts and Approaches to the Study of Prostate Cancer. A.R. Liss: New York, 1987, pp 273-282.

26 Hayward SW, Haughney PC, Rosen MA, et al. Interactions between adult human prostatic epithelium and rat urogenital sinus mesenchyme in a tissue recombination model. Differentiation 1998;63:131-140.

27 Cunha GR, Reese BA, Sekkingstad M. Induction of nuclear androgen-binding sites in epithelium of the embryonic urinary bladder by mesenchyme of the urogenital sinus of embryonic mice. Endocrinology 1980;107:1767-1770.

28 Cunha GR, Lung B, Reese B. Glandular epithelial induction by embryonic mesenchyme in adult bladder epithelium of Balb/c mice. Invest Urol 1980;17: 302-304.

29 Kaplan-Lefko PJ, Chen TM, Ittmann MM, et al. Pathobiology of autochthonous prostate cancer in a pre-clinical transgenic mouse model. Prostate 2003;55: 219-237.

30 Shibata MA, Ward JM, Devor DE, et al. Progression of prostatic intraepithelial neoplasia to invasive carcinoma in C3(1)/SV40 large T antigen transgenic mice: histopathological and molecular biological alterations. Cancer Res 1996;56:4894-4903.

31 Hayward SW. Approaches to modeling stromalepithelial interactions. J Urol 2002;168:1165-1172.

32 Abou-Haila A, Fain-Maurel MA. Regional differences of the proximal part of mouse epididymis: morphological and histochemical characterization. Anat Rec 1984;209:197-208.

33 Tani Y, Suttie A, Flake GP, et al. Epithelial-stromal tumor of the seminal vesicles in the transgenic adenocarcinoma mouse prostate model. Vet Pathol 2005;42:306-314.

34 Jin C, McKeehan K, Wang F. Transgenic mouse with high cre recombinase activity in all prostate lobes, seminal vesicle, and ductus deferens. Prostate 2003;57:160-164.

35 Gao N, Zhang J, Rao MA, et al. The role of hepatocyte nuclear factor-3 alpha (Forkhead Box A1) and androgen receptor in transcriptional regulation of prostatic genes. Mol Endocrinol 2003;17:1484-1507.

Supplementary Information accompanies the paper on Laboratory Investigation website (http://www.nature. com/labinvest) 